\title{
Immobilisation of titanium dioxide onto supporting materials in heterogeneous photocatalysis: a review
}

\begin{abstract}
The aim of this review is to offer an overview of the evolution in the use of different anchors (supports) for the immobilisation of a semiconductor photocatalyst, which is titanium dioxide (TiO2). Several supports and immobilisation techniques that are commonly used for the removal of contaminants in wastewater are discussed. Generally, the immobilisation of a photocatalyst onto supporting material has largely been carried out via one of two major routes; physical (the thermal treatment method) route or chemical (the solï gel method, chemical vapour deposition, electrodeposition, etc.) route. The benefits and drawbacks of various immobilisation techniques to obtain a high surface area $\mathrm{TiO} 2$ support are also discussed.
\end{abstract}

Keyword: Immobilisation; Titanium dioxide; Photocatalyst 